\title{
Gonococcal arthritis complicated by acute pericarditis and pericardial effusion
}

\author{
J Wilson, A G Zaman, A V Simmons
}

\begin{abstract}
A woman of 23 presented with a painful, swollen left knee. A thick yellow fluid aspirated from the joint cavity grew Neisseria gonorrhoeae. Central pleuritic chest pain and ST segment elevation developed 48 hours after presentation. These signs and symptoms of pericarditis had settled two months after the acute illness.
\end{abstract}

We report a case of gonococcal arthritis associated with pericarditis. While on holiday in Southern France, a 23 year old white woman presented to a general hospital with a painful, swollen left knee. Before this, she had complained of a 3 day febrile illness associated with flitting arthralgia affecting the left knee, left elbow, and both shoulders. Clinical examination suggested a septic arthritis of the left knee, and the joint was explored. Thick yellow fluid with a high neutrophil count was aspirated from the joint cavity; a direct smear showed Gram negative intracellular diplococci, and subsequent culture grew Neisseria gonorrhoeae. She was treated with. intravenous ampicillin and bed rest.
Forty eight hours later, she complained of central pleuritic chest pain and was found to have a pericardial rub. An electrocardiogram showed generalised ST segment elevation consistent with the diagnosis of acute pericarditis (figure a), and a chest $x$ ray showed mild cardiomegaly only. A subsequent cross sectional echocardiogram showed a mild to moderate pericardial effusion but no other cardiac abnormality. Her symptoms were treated with aspirin and subsided within 48 hours.

Gynaecological examination was negative and no evidence of genital tract gonorrhoea was detected. The patient's boyfriend was also found to be negative and the patient did not report other sexual contact. The following investigations were found to be either negative or normal; vaginal, rectal, pharyngeal, and urine microbiology; blood culture; Venereal Disease Research Laboratory test; Treponema pallidum haemagluttination assay, hepatitis A and $\mathrm{B}$; rheumatoid and antinuclear factors; antistreptolysin $\mathrm{O}$ and Chlamydia trachomatis titres.

The patient recovered over a period of four weeks, but required two weeks' stay in hospital. The electrocardiogram remained abnor-
Department of Medicine, St James's University Hospital, Leeds

$\mathrm{J}$ Wilson

A G Zaman

A V Simmons

Correspondence to Dr J Wilson, Department of Medicine, G Floor, The Martin Wing, Leeds Genera Infirmary, Great George
Street, Leeds LSI 3EX.

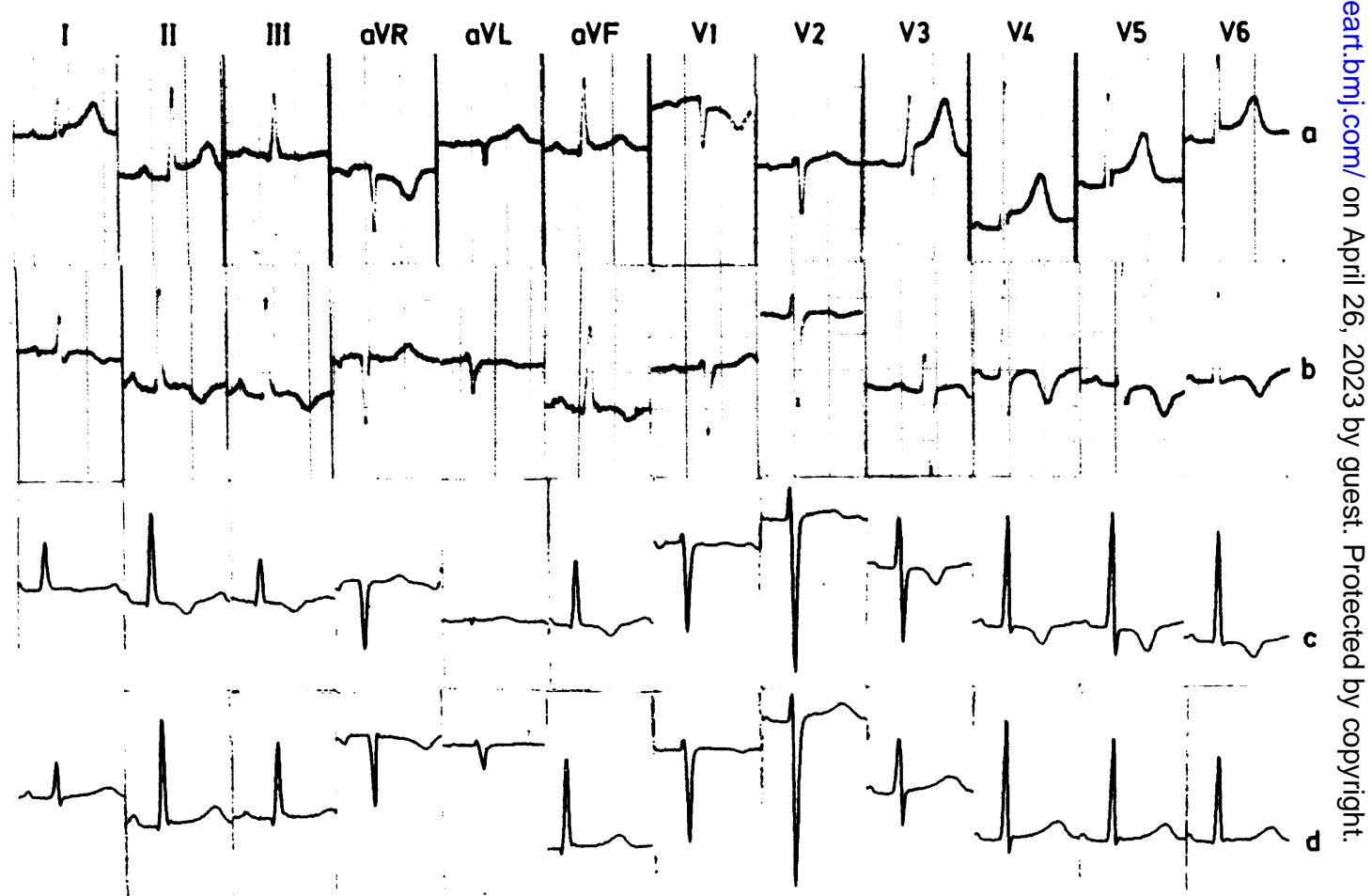

Electrocardiographic tracing showing changes of pericarditis (a) on presentation and (b) one week, (c) six weeks, and (d) two months after presentation. 
mal both 1 week (figure b) and 6 weeks (figure c) after discharge from hospital. A repeat echocardiogram performed 2 months after the illness was normal, and by that time the electrocardiogram had also reverted to normal (figure d). All joint symptoms disappeared completely.

\section{Discussion}

Pericarditis in association with gonococcal arthritis has been infrequently described. ${ }^{1-5}$ In some of these cases the diagnosis of pericarditis was based on an abnormal electrocardiogram rather than clinical presentation, ${ }^{125}$ and this contrasts with our patient who had classic symptoms and signs of the disease. The electrocardiographic abnormalities of pericarditis have been found in disseminated gonococcal infection, ${ }^{25}$ but are only rarely associated with the clinical manifestations, whereas some have found no electrocardiographic abnormalities at all. ${ }^{6}$ Others suspected gonococcal pericarditis but were unable to obtain a positive bacteriological culture..$^{7-10}$

The presence of pericardial effusion has also been infrequently noted, and in Boyle et al's review of 414 cases of purulent pericarditis only two were associated with gonorrhoea. ${ }^{7}$ The pericardial effusion in our patient was only mild to moderate and was not associated with a haemodynamic disturbance, so there was no clinical indication for pericardiocentesis. The nature of the pericardial fluid remains unknown but it did clear after successful treatment of the arthritis, and the follow up echocardiogram was completely normal and showed no evidence of pericardial thickening. Although purulent ${ }^{7}$ and fibrinous ${ }^{3}$ pericarditis have been described, the possibility of an allergic serositis ${ }^{11}$ is not unlikely in our case. We found no published reports of the isolation of the organism from the pericardium, except in the presence of gonococcal endocarditis. Pericardiocentesis can therefore be recommended only for haemodynamic deterioration as a result of tamponade.

The diagnosis in this case was made simple by the positive cultures in the joint cavity, but the differential diagnosis of fever, polyarthralgia, and pericarditis includes diseases such as rheumatoid arthritis, systemic lupus erythematosus, Reiter's syndrome, hepatitis B infection, neoplasm, and other bacterial or viral infections. Primary gonococcal infection is often asymptomatic, ${ }^{2}$ particularly in female patients and the diagnosis and appropriate investigation should be considered in patients presenting with such symptoms.

1 Vietzke WM. Gonococcal arthritis with pericarditis. Arch Intern Med 1966;117:270-2.

2 Holmes KK, Counts GW, Beaty HN. Disseminated gonococcal infection. Ann Intern Med 1971;74:979-93.

3 Robin A, Fiessinger N. Pericardite gonococcique epanchement. Bull Soc Med Hop Paris 1912;34:802-13.

4 Boone JL, Patrone NA, Daugherty JE. Disseminated gonococcal infection and acute pericarditis. NC Med J 1986;47:466-7.

5 Goobar JE, Clark GM. Rheumatological manifestations of gonorrhea. Arch Interamer Rheumatol 1964;7:1-24.

6 Keiser H, Ruben FL, Wollinsky E, et al. Clinical forms of gonococcal arthritis. $N$ Engl J Med 1968;279:234-40.

7 Boyle JD, Pearce ML, Guze LB. Purulent pericarditis: review of literature and report of eleven cases. Medicine 1961;40:119-42.

8 Van der Veer JB, Norris RF. The electrocardiographic changes in acute pericarditis. Am Heart $J$ 1937;14:31-50.

9 Williamson EG. Purulent pericarditis in childhood. An Surg 1927;85:659-67.

10 Thayer WS. On the cardiac complications of gonorrhea Johns Hopkins Hospital Bulletin 1922;33:361-79.

11 O'Brien JP, Goldenberg DL, Rice PA. Disseminated gonococcal infection: a prospective analysis of 49 patients and a review of the pathophysiology and immune mechanisms. Medicine 1983;62:395-406. 